OPEN ACCESS

Edited by:

Mikhail Lebedev,

Duke University, USA

Reviewed by:

Patrizia Silvia Bisiacchi,

University of Padua, Italy

Rachel M. Sherrard,

Pierre-and-Marie-Curie University,

France

*Correspondence:

Dylan J. Edwards

dje2002@med.cornell.edu

Received: 30 March 2016 Accepted: 27 September 2016 Published: 15 November 2016

Citation:

Tsagaris KZ, Labar DR and Edwards DJ (2016) A Framework for Combining rTMS with Behavioral

Therapy.

Front. Syst. Neurosci. 10:82. doi: 10.3389/fnsys.2016.00082

\section{A Framework for Combining rTMS with Behavioral Therapy}

\author{
K. Zoe Tsagaris ${ }^{1}$, Douglas R. Labar ${ }^{1,2}$ and Dylan J. Edwards',2* \\ ${ }^{1}$ Non-Invasive Brain Stimulation and Human Motor Control Laboratory, Burke Medical Research Institute, White Plains, NY, \\ USA, ${ }^{2}$ Department of Neurology, New York Presbyterian, Weill Cornell Medicine, New York, NY, USA
}

Upon its inception, repetitive transcranial magnetic stimulation (rTMS) was delivered at rest, without regard to the potential impact of activity occurring during or around the time of stimulation. rTMS was considered an experimental intervention imposed on the brain; therefore, the myriad features that might suppress or enhance its desired effects had not yet been explored. The field of rTMS has since grown substantially and therapeutic benefits have been reported, albeit with modest and inconsistent improvements. Work in this field accelerated following approval of a psychiatric application (depression), and it is now expanding to other applications and disciplines. In the last decade, experimental enquiry has sought new ways to improve the therapeutic benefits of rTMS, intended to enhance underlying brain reorganization and functional recovery by combining it with behavioral therapy. This concept is appealing, but poorly defined and requires clarity. We provide an overview of how combined rTMS and behavioral therapy has been delineated in the literature, highlighting the diversity of approaches. We outline a framework for study design and reporting such that the effects of this emerging method can be better understood.

\footnotetext{
Keywords: repetitive transcranial magnetic stimulation (rTMS), non-invasive brain stimulation, neurological rehabilitation, behavioral interventions, therapy
}

\section{INTRODUCTION}

The brain is never at rest; the default-mode network comprised of coherent and connected brain networks (cingulate cortices, inferior parietal, and medial prefrontal regions), operative at times of behavioral rest, has been examined through the use of blood oxygenation level-dependent (BOLD) signals. Functional MRI testing has shown the default-mode network is active at rest and in the presence of volitional behavior (e.g., repetitive task practice), decreases in activation. This deactivation may allow for associated activity-dependent plasticity in other parts of the motor system. Because of the default-mode network's activation/deactivation patterns, it appears the motor cortex does not work in isolation during volitional behavior, motor learning, recovery and reorganization (Sanes and Donoghue, 2000; Gusnard and Raichle, 2001; Damoiseaux et al., 2006). Instead, the motor cortex injury-response functional reorganization appears to occur through use-dependent alteration of outputs, which result from behavioral experiences, such as repetitive task practice (Nudo et al., 2001). Interventions promoting recovery should attend to this complex dynamic process, and likely utilize a multi-faceted approach for optimal outcome. 


\section{Background}

The initial application of spatially targeted non-invasive brain stimulation (NIBS) as treatment to improve function was not temporally linked to meaningful voluntary brain activity; instead was delivered in isolation of standard therapy practice techniques (Amassian and Maccabee, 2006). Such applications would seem to tap into only a fraction of the complex multi-faceted systems changes involved in learning and memory. Influencing brain areas remote from, but functionally connected to, a primary target, and influencing brain activity at critical time periods relative to practice and therapy timing, may be important additional considerations in this domain.

Hardwired existing pathways (e.g., skilled movement, speech, or executive function) may have lower threshold for activation by transcranial magnetic stimulation (TMS), and therefore be preferentially predisposed to modulation by NIBS. For example, in the motor system, Rothwell et al. (1987) studied a healthy population at rest and found the largest electromyographic (EMG) responses in distal muscles (which have the finest movements associated with them) versus lower EMG responses in proximal muscles. Furthermore, upon simultaneous voluntary contraction, the EMG response latency to brain TMS shortens, becomes larger, and the threshold is lower. Therefore, if voluntary activation is absent or decreased, such as in a diseased brain state, the modulation possible with NIBS may be altered. Conversely, TMS may affect a patient's ability to produce a voluntary motor activation. Thus the quality and duration of responses seem likely to be influenced by the interactions of interdependent complex systems. The efficacy of standalone NIBS is influenced by its stimulation variables, which include: frequency, intensity, coil positioning, stimulation site, and number of sessions delivered (Nollet et al., 2003; Goetz et al., 2016). Therefore, TMS protocols may be adapted to modulate the motor response through excitatory or depressive methods dependent on stimulation parameters, providing opportunity for custom programs based on the population, person, and pathology.

\section{rTMS and Behavioral Intervention: Current Focus}

One contemporary approach is to link NIBS with behavioral techniques, in hopes of producing more robust and durable outcomes. The logic for doing so stems from the idea that effects of repetitive TMS (rTMS) and behavioral therapy will sum or that rTMS will enhance, or consolidate, the effects of therapy. For instance, Mills and Schubert (1995) suggest TMS during sustained voluntary, tonic activity may increase synchronicity of common input fibers and motor neurons. This improved synchrony may promote increased motor cortex plasticity, compared to one intervention alone. Finding the optimal pairing of intensity, duration, frequency, and site, for both rTMS and therapy, would be important for the strongest enhancement, but also to avoid maladaptive plasticity.

Thus one can learn from analyzing the variety of approaches that have been employed previously and assess outcome. In the present paper we aimed to: (a) outline what is meant by combined therapy in the context of historical literature [this is examining how traditional therapies (occupational, speech, physical, cognitive-behavioral, or task practice) are combined with rTMS either on or off-line]; (b) provide a snapshot of the literature showing the diverse range of approaches, and outcomes; and (c) propose a framework for how combined therapy should be reported in the literature moving forward.

Most combined therapy studies have been on stroke survivors to date and have been intuitively and practically based (Supplementary Table S1). For example, time considerations such as equipment practicality, busy clinical setting, staffing, training, and availability, may affect feasibility of temporally combined therapies. Additionally, the participant's cognitive or physical state during rTMS delivery is rarely reported in the literature but may influence intervention efficacy (e.g., patient engaged in conversation, use of mobile device, listening to music). Despite increasing application of TMS in the field of psychiatry and FDA approval for refractory depression, very little has been done in examining the combination of rTMS with behavioral interventions, such as cognitive behavioral therapy (Micoulaud-Franchi et al., 2013; note French language). Instead, most commonly, papers describe using rTMS in isolation for patients with medication-resistant depression (Lam et al., 2008; Slotema et al., 2010). It would be of considerable interest for systematic research on combined therapy to expand beyond the motor function domain, and study brain processes such as depression or learning in the future. Hopefully this would lead to a better understanding of broader principles concerning the effects of rTMS as an adjunct to older, traditional, well-accepted therapy approaches.

\section{Cumulative Effect}

Research to determine the cumulative effect and duration of results of NIBS intervention is ongoing. This cumulative effect was examined in a healthy population by Baumer et al. (2003) who found repeated sessions of rTMS (two trains of inhibitory, sub-motor threshold rTMS over the pre-motor cortex) delivered within $24 \mathrm{~h}$, or no greater than 7 days later (consecutive days), induced plastic changes of intrinsic motor cortex excitability. Khedr et al. (2006) and Lomarev et al. (2006) found a cumulative effect of rTMS in individuals with Parkinson's Disease (eight sessions of $25 \mathrm{~Hz}$ rTMS over 4 weeks) which lasted 1 month or more. It appears that multiple sessions of rTMS will lead to a cumulative effect, identified as regions of sustained membrane polarization (Pell et al., 2011). With this effect it may be possible to build on neuromodulatory changes in subsequent sessions to promote recovery or slow disease progression. Similarly, in behavioral therapy, efficacy of treatment may be affected by factors including: duration, intensity, type of intervention, and modality used. These therapeutic variables, specifically intensity and duration of therapy, may carry varying levels of importance dependent on individual functional status, though there is still much debate regarding this (Winstein et al., 2016). Thus there are striking similarities between NIBS and traditional therapy variables, in that frequency, intensity, site or system, and duration of the intervention affect outcome. 


\section{LITERATURE SEARCH}

The intent of this search was to identify and summarize published studies combining rTMS with behavioral intervention for clinical benefit. Therefore, we have limited the search to a neurological patient population. All articles were found through the PubMed database and excluded: non-English, case studies, drug studies, transcranial direct current stimulation (tDCS) or other non-invasive stimulation methods, and/or healthy subjects. The acronym rTMS was used in combination with the following search terms (relevant/hits): physical therapy $(23 / 267)$, occupational therapy (8/35), speech therapy (10/54), behavioral intervention (0/95), task training (2/23), motor practice $(3 / 42)$, motor training (3/50), rehabilitation $(8 / 232)$, cognition $(0 / 144)$, cognitive rehabilitation (0/24), cognitive behavioral therapy (2/144), CBT (0/7), cognitive training (0/19). A summary of hits for tDCS in combination with the above search terms is located in Supplementary Figure S1, however, will not be further reviewed in this paper.

\section{LITERATURE ANALYSIS}

The majority of rTMS papers report application of the full stimulation protocol at rest, i.e., not during associated therapy (37/50). A smaller proportion of experimental studies used an interleaved approach of combined therapy, performing the therapeutic task during inter-train intervals (5/50). Less than twenty percent of studies fell into the following categories; unspecified timing (2/50), used high or low frequency dependent on experimental group (3/50), delivered rTMS and therapy simultaneously $(2 / 50)$ or completed rTMS and therapy on different days (1/50).Within these experimental studies, there was great diversity in the stimulation and therapy variables used for each diagnosis within the neurological population (Figure 1). Despite differences, the majority of papers show improvement in clinical outcomes. However, vague and inconsistent reporting of the combined intervention, as well as diverse approaches, impede advancement of understanding toward optimizing intervention and maximizing clinical efficacy. Elements of rTMS application that varied most commonly between studies include; rTMS timing relative to therapy, and either inhibitory (low-frequency), or excitatory (high-frequency) rTMS (Supplementary Table S1). Detailed reporting and standardization for both rTMS and therapy characteristics is a strategy for taking into consideration variations between clinicians, locations, resources, and type of intervention.

\section{Stimulation Targeting}

Of studies investigating chronic motor impairment following stroke (24/50), all but one targeted the primary motor cortex, the exception targeted the somatosensory cortex (Supplementary Table S1, Study \#18). The majority of these studies targeted the unaffected hemisphere (17/24). The remaining studies stimulated the affected hemisphere (5/24; Supplementary Table S1, Study $\# 5,16,18,20,23)$, or stimulated bilaterally (3/24; Supplementary Table S1, Study \#6, 9, 13). With regards to pulse frequency in this population, 14/24 stimulated with low, 7/24 with high (Supplementary Table S1, Study \# 5, 14, 16, 18, 20, 23, 26), and 3/24 alternated between high-frequency stimulation (affected hemisphere) and low-frequency (unaffected hemisphere).

All papers (6/50) addressing motor impairment in a subacute stroke population targeted the primary motor cortex. Three studies stimulated the unaffected hemisphere at low-frequency (Supplementary Table S1, Study \#33, 34, 35), 1 stimulated the affected hemisphere at high-frequency (Supplementary Table S1, Study \#32), and 1 stimulated both hemispheres (unaffected at low-frequency, affected at high-frequency) dependent on treatment group (Supplementary Table S1, Study \#31). Cha and Kim (2016) stimulated the cortical representation of the first right dorsal interosseous muscle at a low-frequency in their study examining unilateral neglect and motor control.

Additional studies in the stroke population were completed in acute stroke [ $>1$ month post onset (Emara et al., 2010)] or in subacute and chronic patients $[3+$ months post onset (Chang et al., 2012)]. In the acute population patients were stimulated at highfrequency (affected motor cortex) or low-frequency (unaffected). Chang et al. (2012) stimulated the affected primary motor cortex at high-frequency. Other motor impairment studies were completed in patients with congenital hemiparesis (children), Parkinson's disease, and hand dystonia (Supplementary Table S1, Study \# 1, 2, 3, respectively). Gillick et al. (2014) stimulated the unaffected primary motor cortex with priming rTMS (high then low-frequency) for children with congenital hemiparesis. Yang et al. (2013) stimulated the contralateral primary motor cortex at high-frequency to "more affected side" in patients with Parkinson's disease. Kimberley et al. (2015) stimulated the unaffected pre-motor cortex at low-frequency in patients with hand dystonia. One study (Lim et al., 2010) studied hemispatial neglect and stimulated the left parietal area (P5; affected side for all participants) at low-frequency.

Studies examining speech impairments following stroke $(n=11)$ were completed in sub-acute ( $<6$ months post-onset; $6 / 11$ ) and chronic populations (>6 months post-onset; 5/11). Within the speech domain, Momosaki et al. (2014) studied oral motor control and stimulated two times daily (low then highfrequency) to the pharyngeal muscles representation. Of studies examining aphasia $(n=10)$, four stimulated Broca's Area (i.e., pars triangularis); 3/4 delivered a low-frequency (Supplementary Table S1, Study \#40, 42, 46), while 1/4 stimulated with priming rTMS [high then low-frequency (Khedr et al., 2014)]. Abo et al. (2012) differentially defined the stimulation target based on aphasia-type, as determined by fMRI activation (inferior frontal gyrus in non-fluent aphasia and superior temporal gyrus in fluent aphasia) and stimulated at low-frequency.

Studies targeting cognitive impairment were completed in Alzheimer's (2/3), and chronic stroke populations (1/3). In the Alzheimer's studies, high-frequency stimulation was delivered to cortical targets including: Broca's Area, Wernicke's Area, right dorsolateral prefrontal cortex, and left parietal somatosensory association cortex (two targets stimulated one day, third target on another) (Supplementary Table S1, Study \#49, 50). Park and Yoon (2015) targeted the left prefrontal cortex in stroke, the affected hemisphere for all participants, at high-frequency. 


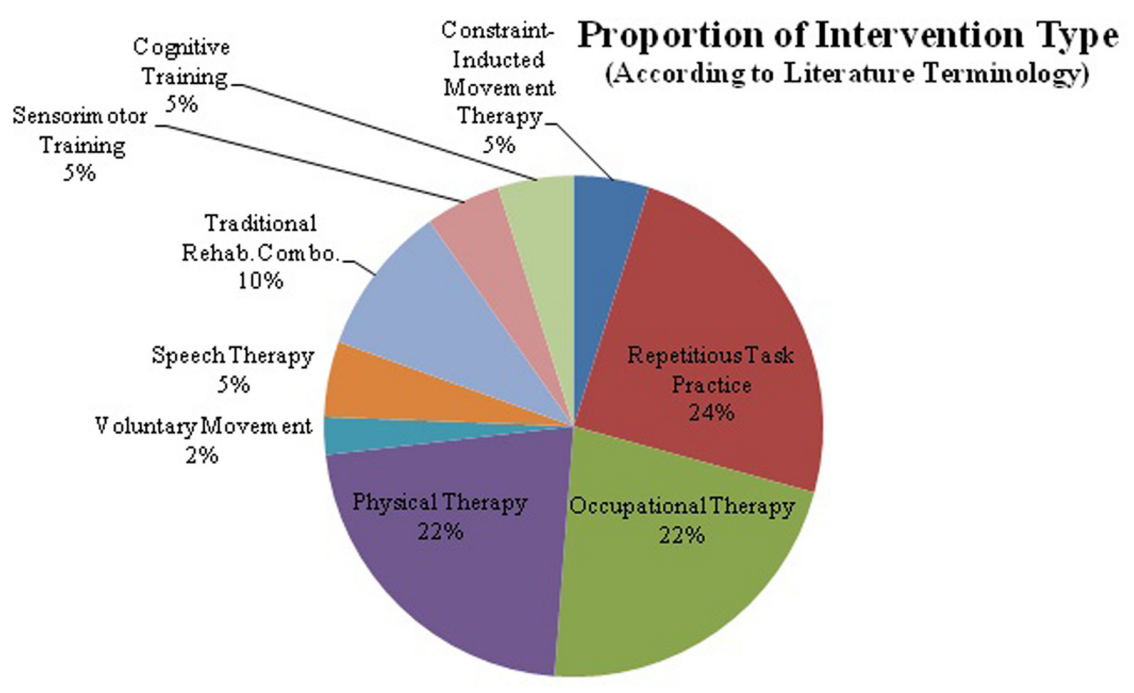

FIGURE 1 | The majority of research in repetitive transcranial magnetic stimulation (rTMS) and behavioral intervention has been completed with rTMS in conjunction with motor training. rTMS paired with speech therapy, cognitive training, and sensorimotor training account for only $30 \%$. rTMS in conjunction with cognitive therapies has not been widely studied, despite FDA approval for use in refractory depression. This graph shows the distribution of behavioral interventions used in conjunction with rTMS. Despite there being a fairly wide variety of interventions utilized there are great differences in protocol design and stimulation parameters, which makes comparing protocols or drawing firm conclusions difficult.

Thus no clearly superior targeting strategy has emerged. Stimulation of a primary lesion area, stimulation of other areas with significant network connections to a primary lesion area, and stimulation of a focal area in the presence of a widespread brain disease process, all seem effective.

\section{Outcomes with Respect to Timing}

The relative timing of combined therapy has not been well explored, and is one of the most poorly reported variables in rTMS studies; however, none of the papers examined in this review reported a negative response, regardless of timing. In papers examining rTMS prior to behavioral intervention, $86.5 \%(32 / 37)$ cited a positive response, while 5/37 (Supplementary Table S1, Study \#3, 20, 28, 35, 42) report a neutral response. The second most frequent approach (5/50) involved alternating protocols (behavioral intervention during the intertrain interval), all of which indicate a positive result in outcome (Supplementary Table S1, Study \#5, 23, 30, 31, 32). Out of the remaining $20 \%$ of identified studies, $7 / 8$ reported a positive response (Supplementary Table S1, Study \#1, 10, 16, 26, 46, 49,50 ), while $1 / 10$ reported a neutral response (Supplementary Table S1, Study \#48). Thus, as in the case of stimulation targeting, discussed above, no clearly superior strategy for temporal pairing of rTMS and behavioral therapy has emerged. However, firm conclusions cannot be drawn due to small sample sizes and need for additional randomized, controlled clinical trials.

For future research and reporting, we propose the temporal relationship of rTMS application with behavioral intervention be defined as: concurrent (rTMS being applied at the same time as the behavior is expressed); sequential (one intervention follows the other), interleaved (rTMS trains are alternating with behavioral expression/repetition) (Figure 2).
One could consider an ongoing pharmacological intervention paired with rTMS a concurrent application of combined therapy, and studies are underway of this nature. Sequential stimulation can occur at numerous time points prior to or following therapy (e.g., volitional activity), ranging from seconds to days. For instance, pulses can be delivered in an event-triggered manner, perhaps with an electroencephalogram (EEG) defined trigger; where important EEG changes known within the neural systems are presently under investigation. Therapeutic rTMS could plausibly be delivered seconds before a task through a clinician prompted visual or verbal cue to promote cognitive effort or movement. Indeed, a single case with positive outcome was reported in the literature for depression where rTMS was combined with a form of cognitive behavioral therapy; here, the cognitive effort was performed in between trains of rTMS so in this case, combined, but alternating (Vedeniapin et al., 2010). Behavioral intervention can be initiated within minutes following stimulation through use of a defined time window to ensure consistency [i.e., therapy starts 5-10 min after rTMS completion, as in clinical trial NCT02089464 (Nexstim Ltd, 2015)]. Stimulation can be delivered within hours or days of therapeutic intervention (i.e., rTMS in morning, therapy in the afternoon or next day). Optimal timing may vary depending on diagnosis and behavioral intervention type, and further research is needed in this area, as $74 \%$ of papers delivered rTMS before behavioral intervention (37/50).

While the physiologic effects of rTMS and behavioral therapy are likely different; a key commonality in both is that after-effects have been linked to adaptive behavioral response, and attributed to lasting modification of synaptic strength in cortical networks subserving the behavior (Butefisch et al., 1995; Silasi and Murphy, 2014), which can be local (e.g., primary motor cortex), or distant 

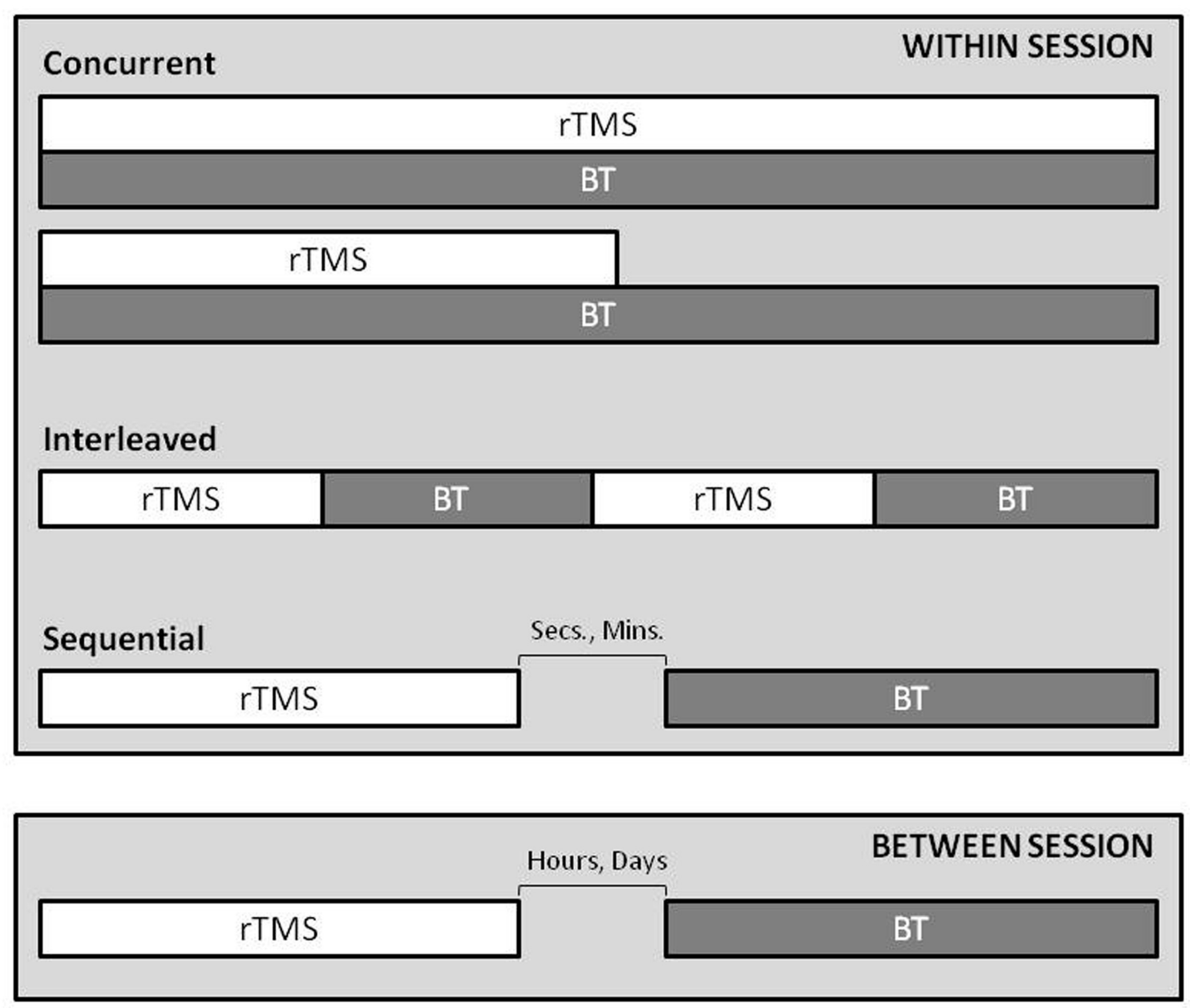

FIGURE 2 | A conceptual guide for timing of rTMS and behavioral therapy. The within session and between session temporal relationship, influences during and after-effect interactions, depending on the time-course of combination. The relationship can be defined as; 1 . concurrent, rTMS is applied at the same time as the behavior is expressed (reporting should include if one intervention outlasts the other), 2. interleaved, rTMS trains are alternating with behavioral

expression/repetition, 3. sequential, one intervention follows the other. Note: Separation of the two techniques by hours or days may not harness the interactions of short term after effects of each.

through functionally connected networks. The physiological interaction of brain stimulation and discrete voluntary behaviors has been well reported experimentally in healthy subjects (Iyer et al., 2003; Daskalakis et al., 2006; Buch et al., 2011), with striking interactive effects, including augmentation, cancelation, and reversal of effect, depending on the circumstances of the interaction. In the context of the present review, the data suggest a more uniform positive response to intervention, which may be troubling. The authors suggest a framework for reporting research methods in studies examining paired rTMS and behavioral interventions (Supplementary Table S2).

\section{rTMS and Behavioral Therapy in Other Functional Domains and Adverse Events}

Currently there is a lack of research on combined applications studying functional domains outside of motor and speech. In the future, rTMS may be a promising approach for increasing the efficacy of currently accepted, traditional behavioral interventions. There were no serious adverse events reported in our reviewed studies, and only mild headaches were reported in a few papers.

\section{CONCLUSION}

Combined therapy of rTMS paired with behavioral intervention has gained traction in the scientific field. Historically, rTMS was delivered in isolation, yet with positive results. Researchers are now examining how rTMS may be used as an adjuvant to more traditional therapies (e.g., physical, occupational, speech, or cognitive therapies) to maximize the benefit of both interventions. To date, the majority of research has 
been completed in the motor-domain of a stroke population and has examined delivering rTMS prior to the behavioral intervention. Of the studies reviewed, combined therapy appears to be safe, since only minor adverse effects (primarily mild headache) were reported in a few papers. Further research is needed to examine the optimal pairing of rTMS and behavioral intervention. Focused attention to all rTMS parameters and timing of stimulation is essential to allow for study replication and data interpretation. Similarly, therapy-related specifications must be clearly reported and standardized. In order to determine optimal combined therapy, larger sample sizes and randomized, controlled clinical trials are needed to account for variability among individuals and conditions.

\section{AUTHOR CONTRIBUTIONS}

KT contributed the acquisition of data and drafting of manuscript. DL and DE contributed study conception and design, as well as critical revision.

\section{REFERENCES}

Abo, M., Kakuda, W., Watanabe, M., Morooka, A., Kawakami, K., and Senoo, A. (2012). Effectiveness of low-frequency rTMS and intensive speech therapy in poststroke patients with aphasia: a pilot study based on evaluation of fMRI in relation to type of aphasia. Eur. Neurol. 68, 199-208. doi: 10.1159/0003 38773

Amassian, V., and Maccabee, P. (2006). “Transcranial magnetic stimulation," in Proceeding of the 28th Annual International Conference of the IEEE Engineering in Medicine and Biology Society, 2006, New York, NY, 1620-1623. doi 10.1109/IEMBS.2006.259398.

Avenanti, A., Coccia, M., Ladavas, E., Provinciali, L., and Ceravolo, M. G. (2012). Low-frequency rTMS promotes use-dependent motor plasticity in chronic stroke: a randomized trial. Neurology 78, 256-264. doi: 10.1212/WNL.0b013e3182436558

Barros Galvao, S., dos Santos, R. B., dos Santos, P., Cabral, M. E., and Monte-Silva, K. (2014). Efficacy of coupling repetitive transcranial magnetic stimulation and physical therapy to reduce upper-limb spasticity in patients with stroke: a randomized controlled trial. Arch. Phys. Med. Rehabil. 95, 222-229. doi: 10.1016/j.apmr.2013.10.023

Baumer, T., Lange, R., Liepert, J., Weiller, C., Siebner, H. R., Rothwell, J. C., et al. (2003). Repeated premotor rTMS leads to cumulative plastic changes of motor cortex excitability in humans. Neuroimage 20, 550-560. doi: 10.1016/S10538119(03)00310-0

Bentwich, J., Dobronevsky, E., Aichenbaum, S., Shorer, R., Peretz, R., Khaigrekht, M., et al. (2011). Beneficial effect of repetitive transcranial magnetic stimulation combined with cognitive training for the treatment of Alzheimer's disease: a proof of concept study. $J$ Neural. Transm. (Vienna) 118, 463-471. doi: 10.1007/s00702-0100578-1

Brodie, S. M., Meehan, S., Borich, M. R., and Boyd, L. A. (2014). $5 \mathrm{~Hz}$ repetitive transcranial magnetic stimulation over the ipsilesional sensory cortex enhances motor learning after stroke. Front. Hum. Neurosci. 8:143. doi: 10.3389/fnhum.2014.00143

Buch, E. R., Johnen, V. M., Nelissen, N., O’Shea, J., and Rushworth, M. F. S. (2011). Noninvasive associative plasticity induction in a corticocortical pathway of the human brain. J. Neurosci. 31, 17669-17679. doi: 10.1523/JNEUROSCI.151311.2011

Butefisch, C., Hummelsheim, H., Denzler, P., and Mauritz, K. (1995). Repetitive training of isolated movements improves the outcome of motor rehabilitation of the centrally paretic hand. J. Neurol. Sci. 130, 59-68. doi: 10.1016/0022510X(95)00003-K

\section{FUNDING}

DE is supported by National Institute of Child Health and Human Development (NICHD) of the NIH (award number R01HD069776).

\section{ACKNOWLEDGMENTS}

The authors would like to acknowledge Daniel Press MD for insight in psychiatric application of rTMS, and Gary Thickbroom Ph.D. for helpful comments during manuscript development.

\section{SUPPLEMENTARY MATERIAL}

The Supplementary Material for this article can be found online at: http://journal.frontiersin.org/article/10.3389/fnsys. 2016.00082/full\#supplementary-material

Cha, H. G., and Kim, M. K. (2016). Effects of repetitive transcranial magnetic stimulation on arm function and decreasing unilateral spatial neglect in subacute stroke: a randomized controlled trial. Clin. Rehabil. 30, 649-656. doi: 10.1177/02692I55I5598817

Chang, W. H., Kim, Y., Bang, O., Kim, S. T., Park, Y. H., and Lee, P. K. W. (2010). Long-term effects of rTMS on motor recovery in patients after subacute stroke. J. Rehabil Med. 42, 758-764. doi: 10.2340/16501977-0590

Chang, W. H., Kim, Y. H., Yoo, W. K., Goo, K. H., Park, C. H., Kim, S. T., et al. (2012). rTMS with motor training modulates cortico-basal gangliathalamocortical circuits in stroke patients. Restor. Neurol. Neurosci. 30, 179189. doi: 10.3233/RNN-2012-110162

Conforto, A. B., Anjos, S. M., Saposnik, G., Mello, E. A., Nagaya, E. M., Santos, W. Jr., et al. (2012). Transcranial magnetic stimulation in mild to severe hemiparesis early after stroke: a proof of principle and novel approach to improve motor function. J. Neurol. 259, 1399-1405. doi: 10.1007/s00415-0116364-7

Cotelli, M., Fertonani, A., Miozzo, A., Rosini, S., Manenti, R., Padovani, A., et al. (2011). Anomia training and brain stimulation in chronic aphasia. Neuropsychol. Rehabil. 21, 717-741. doi: 10.1080/09602011.2011.621275

Damoiseaux, J. S., Rombouts, S., Barkof, F., Scheltens, P., Stam, C., Smith, S., et al. (2006). Consistent resting-state networks across healthy subjects. Proc. Natl. Acad. Sci. U.S.A. 103, 12848-12853. doi: 10.1073/pnas.0601417103

Daskalakis, Z. J., Moller, B., Christensen, B. K., Fitzgerald, P. B., Gunraj, C., and Chen, R. (2006). The effects of repetitive transcranial magnetic stimulation on cortical inhibition in healthy human subjects. Exp. Brain Res. 174, 403-412. doi: 10.1007/s00221-006-0472-0

Emara, T. H., Moustafa, R. R., ElNahas, N. M., ElGanzoury, A. M., Abdo, T. A., Mohamed, S. A., et al. (2010). Repetitive transcranial magnetic stimulation at 1 $\mathrm{Hz}$ and $5 \mathrm{~Hz}$ produces sustained improvement in motor function and disability after ischaemic stroke. Eur. J. Neurol. 17, 1203-1209. doi: 10.1111/j.14681331.2010.03000.x

Gillick, B. T., Krach, L. E., Feyma, T., Rich, T. L., Moberg, K., Thomas, W., et al. (2014). Primed low-frequency repetitive transcranial magnetic stimulation and constraint-induced movement therapy in pediatric hemiparesis: a randomized controlled trial. Dev. Med. Child Neurol. 56, 44-52. doi: 10.1111/dmcn.12243

Goetz, S. M., Luber, B., Lisanby, S. H., Murphy, D. L., Kozyrkov, I. C., Grill, W. M., et al. (2016). Enhancement of neuromodulation with novel pulse shapes generated by controllable pulse parameter transcranial magnetic stimulation. Brain Stimul. 9, 39-47. doi: 10.1016/j.brs.2015.08.013

Gusnard, D. A., and Raichle, M. E. (2001). Searching for a baseline: functional imaging and the resting human brain. Nat. Rev. Neurosci. 2, 685-694. doi: $10.1038 / 35094500$ 
Iyer, M. B., Schleper, N., and Wassermann, E. M. (2003). Priming stimulation enhances depressant effect of low-frequency repetitive transcranial magnetic stimulation. J. Neurosci. 23, 10867-10872.

Kakuda, W., Abo, M., Kobayashi, K., Momosaki, R., Yokoi, A., Fukuda, A., et al. (2010). Low-frequency repetitive transcranial magnetic stimulation and intensive occupational therapy for poststroke patients with upper limb hemiparesis: preliminary study of a 15-day protocol. Int. J. Rehabil. Res. 33, 339-345. doi: 10.1097/MRR.0b013e32833cdf10

Kakuda, W., Abo, M., Kobayashi, K., Momosaki, R., Yokoi, A., Fakuda, A., et al. (2011). Anti-spastic effect of low-frequency rTMS applied with occupational therapy in post-stroke patients with upper limb hemiparesis. Brain Inj. 25, 496-502. doi: 10.3109/02699052.2011.559610

Kakuda, W., Abo, M., Kobayashi, K., Momosaki, R., Yokoi, A., Fukunda, A., et al. (2011). Application of combined 6-Hz primed low frequency rTMS and intensive occupational therapy for upper limb hemiparesis after stroke. Neurorehabilitation 29, 365-371. doi: 10.3233/NRE-2011-0714

Kakuda, W., Abo, M., Kobayashi, K., Takagishi, T., Momosaki, R., Yokoi, A., et al. (2011). Baseline severity of upper limb hemiparesis influences the outcome of low-frequency rTMS combined with intensive occupational therapy in patients who have had a stroke. PM R 3, 516-522. doi: 10.1016/j.pmrj.2011.02.015

Kakuda, W., Abo, M., Momosaki, R., and Morooka, A. (2011). Therapeutic application of $6 \mathrm{~Hz}$ primed low frequency rTMS combined with intensive speech therapy for post-stroke aphasia. Brain Inj. 25, 1242-1248. doi: 10.3109/02699052.2011.608212

Kakuda, W., Abo, M., Nobuyoshi, K., Ishikawa, A., Taguchi, K., and Yokoi, A. (2010). Six-day course of repetitive transcranial magnetic stimulation plus occupational therapy for post-stroke patients with upper limb hemiparesis: a case series study. Disabil. Rehabil. 32, 801-807. doi: 10.3109/09638280903295474

Kakuda, W., Abo, M., Shimizu, M., Sasanuma, J., Okamoto, T., Yokoi, A., et al. (2012). A multi-center study on low-frequency rTMS combined with intensive occupational therapy for upper limb hemiparesis in post-stroke patients. J. Neuroeng. Rehabil. 9:4. doi: 10.1186/1743-0003-9-4

Kakuda, W., Abo, M., Watanabe, S., Momosaki, R., Hashimoto, G., Nakayama, Y., et al. (2013). High-frequency rTMS applied over bilateral leg motor areas combined with mobility training for gait disturbance after stroke: a preliminary study. Brain Inj. 27, 1080-1086. doi: 10.3109/02699052.2013.794973

Khedr, E. M., El-Fetoh, N. A., Ali, A. M., El-Hammady, D. H., Khalifa, H., Atta, H., et al. (2014). Dual-hemisphere repetitive transcranial magnetic stimulation for rehabilitation of poststroke aphasia: a randomized, double-blind clinical trial. Neurorehabil. Neural Repair 28, 740-750. doi: 10.1177/1545968314521009

Khedr, E. M., Rothwell, J. C., Shawky, O. A., Ahmed, M. A., and Hamdy, A. (2006). Effect of daily repetitive transcranial magnetic stimulation on motor performance in Parkinson's disease. Mov. Disord. 21, 2201-2205. doi: $10.1002 / \mathrm{mds} .21089$

Kim, C., Choi, H. E., Jung, H., Lee, B. J., Lee, K. H., and Lim, Y. J. (2014). Comparison of the effects of $1 \mathrm{~Hz}$ and $20 \mathrm{~Hz}$ rTMS on motor recovery in subacute stroke patients. Ann. Rehabil. Med. 38, 585-591. doi: 10.5535/arm.2014.38.5.585

Kim, Y., You, S. H., Ko, M., Park, J., Lee, K., Jang, S. H., et al. (2006). Repetitive transcranial magnetic stimulation-induced corticomotor excitability and associated motor skill acquisition in chronic stroke. Stroke 37, 1471-1476. doi: 10.1161/01.STR.0000221233.55497.51

Kimberley, T. J., Schmidt, R. L. S., Chen, M., Dykstra, D. D., and Buetefisch, C. M. (2015). Mixed effectiveness of rTMS and retraining in the treatment of focal hand dystonia. Front. Hum. Neurosci. 9:385. doi: 10.3389/fnhum.2015. 00385

Koganemaru, S., Sawamoto, N., Aso, T., Sagara, A., Ikkaku, T., Shimada, K., et al. (2015). Task-specific brain reorganization in motor recovery induced by a hybrid-rehabilitation combining training with brain stimulation after stroke. Neurosci. Res. 92, 29-38. doi: 10.1016/j.neures.2014.10.004

Kwon, T. G., Kim, Y., Chang, W., Bang, O., and Shin, Y. (2014). Effective method of combining rTMS and motor training in stroke patients. Restor. Neurol. Neurosci. 32, 223-232. doi: 10.3233/RNN-130313

Lam, R. W., Chan, P., Wilkins-Ho, M., and Yatham, L. (2008). Repetitive transcranial magnetic stimulation for treatment-resistant depression: a systematic review and metaanalysis. Can. J. Psychiatry 53, 621-631.
Lim, J. Y., Kang, E. K., and Paik, N. J. (2010). Repetitive transcranial magnetic stimulation to hemispatial neglect in patients after stroke: an open-label pilot study. J. Rehabil. Med. 42, 447-452. doi: 10.2340/16501977-0553

Lomarev, M. P., Kanchana, S., Bara-Jimenez, W., Iyer, M., Wassermann, E. M., and Hallett, M. (2006). Placebo-controlled study of rTMS for the treatment of Parkinson's disease. Mov. Disord. 21, 325-331. doi: 10.1002/mds.20713

Malcolm, M. P., Triggs, W. J., Light, K. E., Gonzalez, R., Wu, S., Reid, K., et al. (2007). Repetitive transcranial magnetic stimulation as an adjunct to constraintinduced therapy: an exploratory randomized controlled trial. Am. J. Phys. Med. Rehabil. 86, 707-715. doi: 10.1097/PHM.0b013e31813e0de0

Micoulaud-Franchi, J. A., Richieri, R., Lancon, C., and Vion-Dury, J. (2013). [Interactive rTMS protocols in psychiatry]. Encephale 39, 426-431. doi: 10.1016/j.encep.2013.04.009

Mills, K. R., and Schubert, M. (1995). Short term synchronization of human motor units and their responses to transcranial magnetic stimulation. J. Physiol. 483(Pt. 2), 511-523. doi: 10.1113/jphysiol.1995.sp020602

Momosaki, R., Abo, M., and Kakuda, W. (2014). Bilateral repetitive transcranial magnetic stimulation combined with intensive swallowing rehabilitation for chronic stroke dysphagia: a case series study. Case Rep. Neurol. 6, 60-67. doi: $10.1159 / 000360936$

Nexstim Ltd (2015). Niche Trial: Navigated Inhibitory rTMS to Contralesional Hemisphere Trial. Available at: https://clinicaltrials.gov/ct2/show/NCT 02089464 ?term $=$ nexstim\&rank $=1$

Nollet, H., Ham, L. V., Deprez, P., and Vanderstraeten, G. (2003). Transcranial magnetic stimulation: review of the technique, basic principles and applications. Vet. J. 166, 28-42. doi: 10.1016/S1090-0233(03)00025-X

Nudo, R., Plautz, E., and Frost, S. (2001). Role of adaptive plasticity in recovery of function after damage to motor cortex. Muscle Nerve 24, 1000-1019. doi: 10.1002/mus.1104

Park, I., and Yoon, J. (2015). The effect of computer-assisted cognitive rehabilitation and repetitive transcranial magnetic stimulation on cognitive function for stroke patients. J. Phys. Ther. Sci. 27, 773-776. doi: 10.1589/jpts.27.773

Pell, G. S., Roth, Y., and Zangen, A. (2011). Modulation of cortical excitability induced by repetitive transcranial magnetic stimulation: influence of timing in geometrical parameters and underlying mechanisms. Prog. Neurobiol. 93, 59-98. doi: 10.1016/j.pneurobio.2010.10.003

Rabey, J. M., Dobronevsky, E., Aichenbaum, S., Gonen, O., Marton, R. G., and Khaigrekht, M. (2013). Repetitive transcranial magnetic stimulation combined with cognitive training is a safe and effective modality for the treatment of Alzheimer's disease: a randomized, double-blind study. J. Neural Transm. (Vienna) 120, 813-819. doi: 10.1007/s00702-012-0902-Z

Rose, D. K., Patten, C., McGuirk, T. E., Lu, X., and Triggs, W. J. (2014). Does inhibitory repetitive transcranial magnetic stimulation augment functional task practice to improve arm recovery in chronic stroke? Stroke Res. Treat. 2014, 305236. doi: 10.1155/2014/305236

Rothwell, J. C., Thompson, P. D., Day, B. L., Dick, J. P. R., Kachi, T., Cowan, J. M. A., et al. (1987). Motor cortex stimulation in intact man: 1. General characteristics of EMG responses in different muscles. Brain 110(Pt. 5), 11731190. doi: 10.1093/brain/110.5.1173

Rubi-Fessen, I., Hartmann, A., Huber, W., Fimm, B., Rommel, T., Thiel, A., et al. (2015). Add-on effects of repetitive transcranial magnetic stimulation on subacute aphasia therapy: enhanced improvement of functional communication and basic linguistic skills: a randomized controlled study. Arch. Phys. Med. Rehabil. 96, 1935-1944. doi: 10.1016/j.apmr.2015.06.017

Sanes, J. N., and Donoghue, J. P. (2000). Plasticity and primary motor cortex. Annu. Rev. Neurosci. 23, 393-415. doi: 10.1146/annurev.neuro.23.1.393

Seniow, J., Bilik, M., Lesniak, M., Waldowski, K., Iwanski, S., and Czlonkowska, A. (2012). Transcranial magnetic stimulation combined with physiotherapy in rehabilitation of poststroke hemiparesis: a randomized, double blind, placebo-controlled study. Neurorehabil. Neural Repair 26, 1072-1079. doi: $10.1177 / 1545968312445635$

Seniow, J., Waldowski, K., Lesniak, M., Iwanski, S., Czepiel, W., and Czlonkowska, A. (2013). Transcranial magnetic stimulation combined with speech and language training in early aphasia rehabilitation: a randomized double-blind controlled pilot study. Top. Stroke Rehabil. 20, 20-61. doi: $10.1310 /$ tsr2003-250 
Silasi, G., and Murphy, T. H. (2014). Stroke and the connectome: how connectivity guides therapeutic intervention. Neuron 83, 1354-1368. doi: 10.1016/j.neuron.2014.08.052

Slotema, C. W., Blom, J. D., Hoek, H. W., and Sommer, I. E. C. (2010). Should we expand the toolbox of psychiatric treatment methods to include repetitive transcranial magnetic stimulation (rTMS)? A meta-analysis of the efficacy of rTMS in psychiatric disorders. J. Clin. Psychiatry 71, 873-884. doi: 10.4088/JCP.08m04872gre

Takeuchi, N., Tada, T., Toshima, M., Chuma, T., Matsuo, Y., and Ikoma, K. (2008). Inhibition of the unaffected motor cortex by $1 \mathrm{~Hz}$ repetitive transcranial magnetic stimulation enhances motor performance and training effect of the paretic hand in patients with chronic stroke. J. Rehabil. Med. 40, 298-303. doi: 10.2340/16501977-0181

Thiel, A., Hartmann, A., Rubi-Fessen, I., Anglade, C., Kracht, L., Weiduschat, N., et al. (2013). Effects of noninvasive brain stimulation on language networks and recovery in early poststroke aphasia. Stroke 44, 2240-2246. doi: 10.1161/STROKEAHA.111.000574

Vedeniapin, A., Cheng, L., and George, M. S. (2010). Feasibility of simultaneous cognitive behavioral therapy (CBT) and left prefrontal rTMS for treatment resistant depression. Brain Stimul. 3, 207-210. doi: 10.1016/j.brs.2010. 03.005

Vongvaivanichakul, P., Tretriluxana, J., Bovonsunthonchai, S., Pakaprot, N., and Laksanakorn, W. (2014). Reach-to-grasp training in individuals with chronic stroke augmented by low-frequency repetitive transcranial magnetic stimulation. J. Med. Assoc. Thai. 97(Suppl. 7), S45-S49.

Waldowski, K., Seniow, J., Lesniak, M., Iwanski, S., and Czlonkowska, A. (2012). Effect of low-frequency repetitive transcranial magnetic stimulation on naming abilities in early-stroke aphasic patients: a prospective, randomized, double-blind sham-controlled study. Sci. World J. 2012: 518568. doi: 10.1100/2012/518568

Wang, C., Hsieh, C., Tsai, P., Wang, C., Lin, F., and Chan, R. (2014). Efficacy of synchronous verbal training during repetitive transcranial magnetic stimulation in patients with chronic aphasia. Stroke 45, 3656-3662. doi: 10.1161/STROKEAHA.114.007058

Wang, R., Tseng, H., Liao, K., Wang, C., Lai, K., and Yang, Y. (2012). rTMS combined with task-oriented training to improve symmetry of interhemispheric corticomotor excitability and gait performance after stroke: a randomized trial. Neurorehabil. Neural Repair 26, 222-230. doi: $10.1177 / 1545968311423265$

Weiduschat, N., Thiel, A., Rubi-Fessen, I., Hartmann, A., Kessler, J., Merl, P., et al. (2011). Effects of repetitive transcranial magnetic stimulation in aphasic stroke: a randomized controlled pilot study. Stroke 42, 409-415. doi: 10.1161/STROKEAHA.110.597864

Winstein, C. J., Wolf, S. L., Dromerick, A. W., Lane, C. J., Nelsen, M. A., Lewthwaite, R., et al. (2016). Effect of task-oriented rehabilitation program on upper extremity recovery following motor stroke: the ICARE randomized clinical trial. JAMA 315, 571-581. doi: 10.1001/jama.2016. 0276

Yamada, N., Kakuda, W., Kondo, T., Shimizu, M., Mitani, S., and Abo, M. (2013). Bihemispheric repetitive transcranial magnetic stimulation combined with intensive occupational therapy for upper limb hemiparesis after stroke: a preliminary study. Int. J. Rehabil. Res. 36, 323-329. doi: 10.1097/MRR.0b013e3283624907

Yang, Y., Tseng, C., Chiou, S., Liao, K., Cheng, S., Lai, K., et al. (2013). Combination of rTMS and treadmill modulates corticomotor inhibition and improves walking in Parkinson Disease: A Randomized Trial. Neurorehabil. Neural Repair 27, 79-86. doi: 10.1177/1545968312451915

Zheng, C., Liao, W., and Xia, W. (2015). Effect of combined low-frequency rTMS and virtual reality training on upper limb function in subacute stroke: a doubleblind randomized controlled trial. J. Huazhong Univ. Sci. Technolog. Med. Sci. 35, 248-254. doi: 10.1007/s11596-015-1419-0

Conflict of Interest Statement: The authors declare that the research was conducted in the absence of any commercial or financial relationships that could be construed as a potential conflict of interest.

Copyright (c) 2016 Tsagaris, Labar and Edwards. This is an open-access article distributed under the terms of the Creative Commons Attribution License (CC BY). The use, distribution or reproduction in other forums is permitted, provided the original author(s) or licensor are credited and that the original publication in this journal is cited, in accordance with accepted academic practice. No use, distribution or reproduction is permitted which does not comply with these terms. 